\title{
Coupling of head and hand movements during eye-head-hand coordination: there is more to reaching than meets eye
}

\author{
K. Hadjidimitrakis \\ Department of Biomedical and Neuromotor Sciences, University of Bologna, Bologna, Italy
}

\begin{abstract}
Hadjidimitrakis K. Coupling of head and hand movements during eye-head-hand coordination: there is more to reaching than meets eye. J Neurophysiol 123: 000 - 000, 2020. First published April 1, 2020; doi:10.1152/jn.00099.2020.-Does arm reaching affect eye-head shifts? Does the head alter eye-hand coordinated movements? Sensorimotor research has focused on either eye-head or eye-hand coordination, with only occasional works studying all these effectors together. Arora et al. (Arora HK, Bharmauria V, Yan X, Sun S, Wang H, Crawford JD. J Neurophysiol 122: 1946 -1961, 2019) examined eyehead-hand coordination for the first time in nonhuman primates and provide evidence suggesting that head and hand movements are more coupled than traditionally considered.
\end{abstract}

arm movement; depth; eye-hand coordination; eye-head coordination; gaze

An important question of sensorimotor neuroscience is to unravel how the brain controls multiple effectors to produce coordinated movements. Since the pioneering studies of Bizzi et al. (1971) and Prablanc et al. (1979) on eye-head and eyehand coordination, respectively, these two types of move- ment have been a model system for understanding how the brain controls multisegmental motion. A huge amount of studies, both in humans and in nonhuman primates, were focused on either of these systems.

The coordinated movements of the eyes and head that enable high spatial frequency samples of the visual environment by shifting the line of sight (gaze) from one part of the visual scene to another are an essential aspect of the visual process. Eyehead coordinated movements are quite stereotyped: they typically start with a rapid eye movement (saccade) that is followed by a slower head movement in the same direction. In the last phase, the vestibulo-ocular reflex stabilizes the gaze by moving the eyes in the opposite direction till the head stops moving. In monkeys and humans there is a high degree of variability in the relative timing of eye and head movements (Guitton and Volle 1987). The relative contribution of ocular and head components to gaze shifts depends on the species, the subject, the eccentricity of the target and the initial position of the eyes (Freedman and Sparks 1997). The variability on this pattern is expressed by the variable time difference between the onset of eye and head movements and their relative contribution (Freedman and Sparks 1997; Sparks et al. 2001).

Address for correspondence: K. Hadjidimitrakis, Department of Biomedical and Neuromotor Sciences, University of Bologna, Piazza di Porta San Donato 2, Bologna 40126, Italy (e-mail: kon.chatzidimitrakis@unibo.it).
Like eye-head coordination, eye-hand coordination is a fundamental behavior that primates use to interact with the world. When humans reach to grasp objects, they look at the target first, then bring the hand to the center of gaze as the object is grasped (Hayhoe et al. 2002; Johansson et al. 2001; Land et al. 1999). Nonhuman primates use the same strategy (Dean et al. 2011; Song and McPeek 2009). Foveation of target before reach onset has been shown to improve hand movement accu- racy (Biguer et al. 1982; Prablanc et al. 1979). During eye- hand coordinated movements, reaction times of saccades and reaches are significantly correlated in both humans and non- human primates (Biguer et al. 1982; Dean et al. 2011; Suzuki et al. 2008; Yttri et al. 2013). Furthermore, in monkeys saccade and reach end points are significantly correlated (Kattoulas et al. 2008) and the presence of a reach reduces saccadic latency (Snyder et al. 2002), thus suggesting a common controlling mechanism.

What happens when all three effectors i.e., eye, head, and hand move simultaneously and need to be coordinated? Arora et al. (2019) were the first to address this question in nonhuman primates. In their study, two macaque monkeys were seated in front of a touchscreen where a visual target was presented in one of eight peripheral locations that covered a large part of the visual field. In the reach task the animals had to perform both a gaze shift from a central screen position to the peripheral target location, and a forward reach to move their hand from a rest position next to their waist and touch the same target. In the gaze shift task monkeys only shifted their gaze and looked at the peripheral target without performing a reach. In both tasks, Arora and colleagues (2019) examined both spatial and temporal relationships and the kinematics of eye, head, and hand movements. The authors reported a number of interesting findings. First, head movements were larger in the reach compared with the gaze shift task, especially along the vertical axis. Despite this, gaze precision was higher in the former task and this was due to a smaller range of eye-in-head positions. Second, regarding the temporal relationships between the effectors, gaze shifts ended just before the onset of the hand movement and gaze remained stable till the hand touched the target. At the same time, although the head movement started during the gaze shift in both tasks, in the reach trials its largest component was executed after the gaze shift ended and this late component occurred mostly along the vertical direction. Third, regarding movement kinematics, peak head velocity for a given head movement amplitude was higher during the reach 
task and, more importantly, the mean hand velocity was much more strongly correlated with peak head velocity compared with peak gaze velocity.

Altogether, the results of Arora et al. (2019) suggest a stronger coupling of head and arm with respect to the eye that seems rather unexpected. However, a significant number of older - but also some recent - human studies are in line with their findings. Smeets et al. (1996) tested the effect of hand movement on eye-head coordination under various conditions of a naturalistic object manipulation task and found in all of them strong correlation between head and hand reaction times and peak velocities. To explain their results, these authors based on evidence from Bizzi et al. (1971), suggesting that the predictability of target position is the most important factor affecting eye-head coordination. In other words, the more predictable is the target of interest, the more the head would be decoupled from the gaze shift. Accordingly, Smeets et al. 1996 argued that head movement facilitates subsequent gaze shifts toward the future position of the hand to guide object manipulations, thus leading to strong correlation between head and hand movement parameters. In the same line Pelz et al. (2001), using a similar task paradigm with Smeets and colleagues, found that the head showed a considerable degree of independence from the gaze and often head movements followed hand trajectory.

This last finding could account for one of the main findings reported by Arora and colleagues (2019), i.e., that the major component of head movement during the reaching task occurred mostly along the vertical axis and was coincident with hand movement. In fact, although Arora et al. (2019) did not mention it explicitly, it is evident that all reach trajectories in their study had a large upward component (see their Fig. 3, C and $D$ ) and this could account for the vertical head movement that occurred after the gaze shift ended, simultaneously with the hand movement. Adding further support to this interpreta- tion of Arora et al. (2019) findings, very recent evidence by Berger et al. (2020) has shown that in freely moving monkeys performing either reaches, or walk-to-reach movement sequences, the activity in frontal and parietal cortex was correlated not only with hand kinematics, but also with translational head movements. The experiments by Berger et al. (2020) highlight the fact that the classic approach of constraining movement in a single or in a couple of joints that has been widely used in neurophysiological studies of motor control to facilitate data analysis and results interpretation should be revised and novel task paradigms using reaching movements under whole body motion in various behavioral constrains should be used. The advent of wireless, multielectrode arrays that can record neural activity from several brain regions, combined with simultaneous motion capture recordings from multiple effectors, makes such methodological approaches entirely feasible today. Such an approach could provide a more comprehensive view of the neural substrates of motor encoding in the parietofrontal network under more natural behavioral contexts.

Such natural behavioral contexts have been employed in an increasing number of recent human studies that also provide further support to Arora et al. (2019) findings. The effect of hand movements on eye-head shifts performed under different whole body postures (e.g., standing, sitting) was examined in one work where larger head movements were reported when the hand moved that resulted in final head position being aligned with the target (Stamenkovic et al. 2018). The authors argued that the extra head displacement could facilitate the underlying sensorimotor transformations from a visual/eyecentered to a proprioceptive/hand-centered reference frame that are necessary for the planning of hand movement. In a similar vein, Arora et al. (2019) propose that the additional head movement that brings the target at or near the fovea could be a mechanism for optimizing depth vision for the last phase of reach movement when the hand is about to touch the target. These two speculations are not mutually exclusive and it should be noted that both of them suggest that, in the presence of a hand movement, gaze shifts are being adapted to optimize reaching.

Nevertheless, the influence of hand movement in depth on facilitating the coordination between hand and head movements needs to be studied further. For example, it would be interesting to examine to what extent the fact that in Arora et al. (2019) the eyes and the hand moved along somewhat different parts of space (touchscreen versus 3D space) contributed to the stronger relationship between hand and head with respect to eye. Regarding this issue, another recent human study (Reppert et al. 2018) that investigated the rigor (peak velocity for a given amplitude) of eye, head, and hand movements when constrained in 2D space found somewhat different results with respect to Arora et al. (2019). In that study the eye started moving first and was followed by the hand and then the head. In addition, peak head velocities were lower during combined gaze shifts and reaches compared with when only gaze shifts occurred (Reppert et al. 2018). Despite these differences, Reppert and colleagues (2018) found the head and hand movement vigor to be significantly correlated, whereas their correlation with the vigor of eye movement was weak and not significant.

This coupling of head and hand movements suggests some common neural pathways. While the neural substrates of eye-head and eye-hand coordination have been extensively studied (for references see Andersen et al. 2014; Sparks et al. 2001), the circuits that are responsible for head movement control are less studied and even more so the potential substrates of head-hand coordination. Arora et al. (2019) propose several brain areas involved in head-hand coordination, namely the superior colliculus (SC) in the brainstem, the frontal eye fields (FEF), and the parietal reach region (PRR) in the frontal and parietal cortex, respectively. The SC is well established as a key node of the gaze orienting subcortical circuits, and electrical stimulation of the intermediate and deeper SC layers produces combinations of eye and head movements in the monkey (Cowie and Robinson 1994; Freedman et al. 1996). Furthermore, there is evidence of arm movement related activity in a more restricted zone of SC (Werner et al. 1997), so the convergence of head and hand signals could take place in SC. Regarding the cortex, apart from the well known role of FEF in controlling gaze shifts (e.g., Tu and Keating 2000), there is some evidence for independent head control (Chen 2006) in this area. FEF are located next to the dorsal premotor cortex (PMd), where neurons with oculomotor and hand move- ment activity have been identified (Fujii et al. 2000). Lastly, Arora and colleagues (2019) suggest that also the parietal cortex could implement multieffector coordination, given its significant contribution in coordinating saccades with hand movements, but also speculate that larger parietofrontal circuits could be involved (Andersen et al. 2014; Yttri et al. 2013). 
Departing from this last point, there is quite compelling evidence for the involvement of two other regions in the headhand coordination, one in parietal and the other in frontal cortex. In the former lies the ventral intraparietal area (VIP) that has been reported to control head movements and head- hand synergies occurring during defensive-like behaviors, i.e., the hand moving to protect the head (Cooke et al. 2003). Interestingly, VIP encodes the peripersonal space and has both visual and tactile receptive around the head (Schlack et al. 2005) and strongly projects to a small part of the ventral premotor cortex (PMv) with very similar sensory and motor properties, the polysensory zone (PZ, Graziano et al. 2002). In the frontal cortex, area PMv could be involved in the head-hand coordination. In fact, apart from defensive-like move- ments, the head and hand move in a coordinated manner also during feeding. Together with controlling head orientation, feeding requires the grasping of food. Given that, it is not surprising that Borra et al. (2010) found that the hand region of the PMv - a key grasping area-projects to not only to the SC zone where arm related activity was reported (Werner et al. 1997), but also to the upper cervical spinal cord where the motor neurons innervating the neck muscles are located.

In sum, another interpretation of Arora et al.'s (2019) findings could be based on the activation of VIP and PMv circuits that support the basic motor repertoire of protecting the head during navigation and of coordinating the hand with the head during feeding. It is worthwhile recording simultaneously from both areas during a large variety of naturalistic head-hand coordinated movements and also investigating whether deactivating either of these areas or both disrupts neural processes that couple the two effectors.

\section{GRANTS}

This research was supported by Ministero dell'Istruzione, dell'Università e della Ricerca (Ministry of Education, Research and Universities): PRIN2017KZNZLN; Fondazione Cassa di Risparmio in Bologna: 2018/ 0373; and EC Horizon 2020 (EU Framework Programme for Research and Innovation): MSCA-734227-PLATYPUS.

\section{DISCLOSURES}

No conflicts of interest, financial or otherwise, are declared by the authors.

\section{AUTHOR CONTRIBUTIONS}

K.H. drafted manuscript; K.H. edited and revised manuscript; K.H. approved final version of manuscript.

\section{REFERENCES}

Andersen RA, Andersen KN, Hwang EJ, Hauschild M. Optic ataxia: from Balint's syndrome to the parietal reach region. Neuron 81: 967-983, 2014. doi:10.1016/j.neuron.2014.02.025

Arora HK, Bharmauria V, Yan X, Sun S, Wang H, Crawford JD. Eyehead-hand coordination during visually guided reaches in head-unre- strained macaques. J Neurophysiol 122: 1946 -1961, 2019. doi:10.1152/jn. 00072.2019

Berger M, Agha N, Gail A. Wireless recording from unrestrained monkeys reveals motor goal encoding beyond immediate reach in frontoparietal cortex (Preprint). bioRxiv 305334, 2020. doi:10.1101/305334.

Biguer B, Jeannerod M, Prablanc C. The coordination of eye, head, and arm movements during reaching at a single visual target. Exp Brain Res 46: 301304, 1982. doi:10.1007/BF00237188.
Bizzi E, Kalil RE, Tagliasco V. Eye-head coordination in monkeys: evidence for centrally patterned organization. Science 173: 452-454, 1971. doi:10. 1126/science.173.3995.452.

Borra E, Belmalih A, Gerbella M, Rozzi S, Luppino G. Projections of the hand field of the macaque ventral premotor area F5 to the brainstem and spinal cord. J Comp Neurol 518: 2570 -2591, 2010. doi:10.1002/cne.22353.

Chen LL. Head movements evoked by electrical stimulation in the frontal eye field of the monkey: evidence for independent eye and head control. $J$ Neurophysiol 95: 3528 -3542, 2006. doi:10.1152/jn.01320.2005.

Cooke DF, Taylor CSR, Moore T, Graziano MSA. Complex movements evoked by microstimulation of the ventral intraparietal area. Proc Natl Acad Sci USA 100: 6163-6168, 2003. doi:10.1073/pnas.1031751100.

Cowie RJ, Robinson DL. Subcortical contributions to head movements in macaques. I. Contrasting effects of electrical stimulation of a medial pontomedullary region and the superior colliculus. J Neurophysiol 72: 2648 2664, 1994. doi:10.1152/jn.1994.72.6.2648.

Dean HL, Martí D, Tsui E, Rinzel J, Pesaran B. Reaction time correlations during eye-hand coordination: behavior and modeling. J Neurosci 31: 2399 -2412, 2011. doi:10.1523/JNEUROSCI.4591-10.2011.

Freedman EG, Sparks DL. Eye-head coordination during head-unrestrained gaze shifts in rhesus monkeys. J Neurophysiol 77: $2328-2348,1997$. doi:10.1152/jn.1997.77.5.2328.

Freedman EG, Stanford TR, Sparks DL. Combined eye-head gaze shifts produced by electrical stimulation of the superior colliculus in rhesus monkeys. J Neurophysiol 76: 927-952, 1996. doi:10.1152/jn.1996.76.2.927.

Fujii N, Mushiake H, Tanji J. Rostrocaudal distinction of the dorsal premotor area based on oculomotor involvement. J Neurophysiol 83: $1764-1769$, 2000. doi:10.1152/jn.2000.83.3.1764.

Graziano MSA, Taylor CSR, Moore T. Complex movements evoked by microstimulation of precentral cortex. Neuron 34: 841-851, 2002. doi:10. 1016/S0896-6273(02)00698-0.

Guitton D, Volle M. Gaze control in humans: eye-head coordination during orienting movements to targets within and beyond the oculomotor range. $J$ Neurophysiol 58: 427-459, 1987. doi:10.1152/jn.1987.58.3.427.

Hayhoe M, Aivar P, Shrivastavah A, Mruczek R. Visual short-term memory and motor planning. Prog Brain Res 140: 349 -363, 2002. doi:10.1016/ S0079-6123(02)40062-3.

Johansson RS, Westling G, Bäckström A, Flanagan JR. Eye-hand coordination in object manipulation. J Neurosci 21: 6917-6932, 2001. doi:10. 1523/JNEUROSCI.21-17-06917.2001.

Kattoulas E, Smyrnis N, Mantas A, Evdokimidis I, Raos V, Moschovakis A. Arm movement metrics influence saccade metrics when looking and pointing towards a memorized target location. Exp Brain Res 189: 323-338, 2008. doi:10.1007/s00221-008-1427-4.

Land M, Mennie N, Rusted J. The roles of vision and eye movements in the control of activities of daily living. Perception 28: 1311-1328, 1999. doi:10.1068/p2935.

Pelz J, Hayhoe M, Loeber R. The coordination of eye, head, and hand movements in a natural task. Exp Brain Res 139: 266 -277, 2001. doi:10. 1007/s002210100745.

Prablanc C, Echallier JF, Komilis E, Jeannerod M. Optimal response of eye and hand motor systems in pointing at a visual target. I. Spatio-temporal characteristics of eye and hand movements and their relationships when varying the amount of visual information. Biol Cybern 35: 113-124, 1979. doi:10.1007/BF00337436.

Reppert TR, Rigas I, Herzfeld DJ, Sedaghat-Nejad E, Komogortsev O, Shadmehr R. Movement vigor as a traitlike attribute of individuality. $J$ Neurophysiol 120: 741-757, 2018. doi:10.1152/jn.00033.2018.

Schlack A, Sterbing-D'Angelo SJ, Hartung K, Hoffmann KP, Bremmer F. Multisensory space representations in the macaque ventral intraparietal area. J Neurosci 25: 4616 - 4625, 2005. doi:10.1523/JNEUROSCI.0455-05.2005.

Smeets JBJ, Hayhoe MM, Ballard DH. Goal-directed arm movements change eye-head coordination. Exp Brain Res 109: 434 - 440, 1996. doi:10. 1007/BF00229627.

Snyder LH, Calton JL, Dickinson AR, Lawrence BM. Eye-hand coordination: saccades are faster when accompanied by a coordinated arm movement. J Neurophysiol 87: 2279 -2286, 2002. doi:10.1152/jn.00854.2001.

Song J-H, McPeek RM. Eye-hand coordination during target selection in a pop-out visual search. J Neurophysiol 102: 2681-2692, 2009. doi:10.1152/ jn.91352.2008.

Sparks DL, Freedman EG, Chen LL, Gandhi NJ. Cortical and subcortical contributions to coordinated eye and head movements. Vision Res 41: 3295 3305, 2001. doi:10.1016/S0042-6989(01)00063-3. 
Stamenkovic A, Stapley PJ, Robins R, Hollands MA. Do postural constraints affect eye, head, and arm coordination? J Neurophysiol 120: 2066 2082, 2018. doi:10.1152/jn.00200.2018.

Suzuki M, Izawa A, Takahashi K, Yamazaki Y. The coordination of eye, head, and arm movements during rapid gaze orienting and arm pointing. Exp Brain Res 184: 579 -585, 2008. doi:10.1007/s00221-007-1222-7.

Tu TA, Keating EG. Electrical stimulation of the frontal eye field in a monkey produces combined eye and head movements. J Neurophysiol 84: 11031106, 2000. doi:10.1152/jn.2000.84.2.1103.
Werner W, Dannenberg S, Hoffmann KP. Arm-movement-related neurons in the primate superior colliculus and underlying reticular formation: comparison of neuronal activity with EMGs of muscles of the shoulder, arm and trunk during reaching. Exp Brain Res 115: 191-205, 1997. doi:10.1007/ PL00005690.

Yttri EA, Liu Y, Snyder LH. Lesions of cortical area LIP affect reach onset only when the reach is accompanied by a saccade, revealing an active eyehand coordination circuit. Proc Natl Acad Sci USA 110: 2371-2376, 2013. doi:10.1073/pnas. 1220508110 